\title{
Vibração e ruído no posto de operação de um trator cafeeiro acoplado a um turbo pulverizador sob diferentes velocidades de trabalho
}

\section{Vibration and noise at the operating station of a coffee tractor coupled to a turbo sprayer under different working speeds}

\author{
Geraldo Gomes de Oliveira Júnior ${ }^{1 *}$, Irlon de Angelo da Cunha ${ }^{2}$, José Antônio Ramos da \\ Silva $^{1}$, Lucas Deleon Ramirio ${ }^{1}$, Adriano Bortolotti da Silva ${ }^{3}$, Fernando Ferrari Putti ${ }^{4}$
}

\section{RESUMO}

O presente estudo teve como objetivo determinar o nível de vibração de corpo inteiro (VCI) e ruído de um trator cafeeiro acoplado a um turbo pulverizador em diferentes velocidades de trabalho. O estudo foi conduzido no Instituto Federal de Educação, Ciência e Tecnologia do Sul de Minas - campus Muzambinho. $\mathrm{O}$ experimento foi em Delineamento Inteiramente Casualizado (DIC), com o conjunto mecanizado deslocando-se em 4 diferentes velocidades $\left(2,3 ; 3,5 ; 4,6 ; 5,8 \mathrm{Km} \mathrm{h}^{-1}\right)$. A quantificação dos níveis de VCI foi realizada por medidor de vibração ajustado de acordo com a Norma de Higiene Ocupacional - NHO 09 e para a avaliação do ruído, dosímetro configurado de acordo com a NR 15 e NHO 01. Os resultados mostraram que o aumento da velocidade contribuiu para elevar os níveis de VCI. Nas velocidades de 4,6 e $5,8 \mathrm{Km} \mathrm{h}^{-1}$ os valores de aceleração resultante de exposição normalizada (aren) e valor da dose de vibração resultante (VDVR) ultrapassaram o nível de ação (NR 9), mas ficaram abaixo do limite de exposição (NR 15). A velocidade não influenciou significativamente para os níveis de ruído, no entanto em todas as velocidades avaliadas o ruído ficou acima do limite de exposição de $85 \mathrm{~dB}$ (A) para 8 horas de trabalho.

Palavras-chave: Agentes de riscos físicos; Conjunto mecanizado; Exposição ocupacional;

\section{ABSTRACT}

The present study aimed to determine the level of whole body vibration (WBV) and noise of a coffee tractor coupled to a turbo sprayer at different working speeds. The study was conducted at the Federal Institute of Education, Science and Technology of the South of Minas - Muzambinho. The experiment was carried out in a completely randomized design (CRD), with the mechanized set moving at 4 different speeds $(2.3 ; 3.5$; 4.6; $5.8 \mathrm{~km} \mathrm{~h}^{-1}$ ). The quantification of WBV levels was performed by a vibration meter adjusted in accordance with the Occupational Hygiene Standard - NHO 09 and for noise assessment, a dosimeter configured in accordance with NR 15 and NHO 01. The results showed that the increase in speed contributed to raising the levels of WBV. At speeds of 4.6 and $5.8 \mathrm{~km} \mathrm{~h}^{-1}$, the acceleration values resulting from normalized exposure (arne) and the resulting vibration dose value (RVDV) exceeded the action level (NR 9), but were below the limit exposure (NR 15). Speed did not significantly influence noise levels, however, at all speeds evaluated, noise was above the exposure limit of $85 \mathrm{~dB}$ (A) for 8 hours of work.

Keywords: Physical risk agentes; Mechanized set; Occupational exposure;

\footnotetext{
${ }^{1}$ Instituto Federal de Educação, Ciência e Tecnologia do Sul de Minas - IFSULDEMINAS, campus Muzambinho - MG. *E-mail: geraldo.junior@muz.ifsuldeminas.edu.br

${ }^{2}$ Tecnologista [aposentado] Fundação Jorge Duprat e Figueiredo - FUNDACENTRO

${ }^{3}$ Universidade José do Rosário Vellano - UNIFENAS, campus Alfenas - MG

${ }^{4}$ Universidade Estadual Paulista - UNESP, campus Tupã - SP.
} 


\section{INTRODUÇÃO}

Ao longo do tempo, a cafeicultura vem passando por diversas mudanças, especialmente relacionada a mecanização dos tratos culturais que anteriormente eram realizados manualmente (SILVA et al., 2018). Grande parte deste processo de mecanização se deve ao desenvolvimento e evolução tecnológica dos tratores cafeeiros. De acordo com Santos et al (2016), os tratores são fundamentais para aumento da produtividade e eficiência no uso de insumos, bem como redução dos custos de produção.

No entanto, durante suas atividades, operadores de tratores agrícolas estão expostos a riscos ocupacionais que tem potencial de trazer danos a sua saúde, tais como níveis elevados de ruído e vibrações de corpo inteiro (VCI). Leite et al (2020) afirmaram que é fundamental a quantificação destes agentes de riscos de modo a verificar se os mesmos estão dentro dos limites de exposição estabelecidos pela legislação vigente.

No Brasil, a Norma Regulamentadora NR 15 estabelece como limite de exposição ocupacional ao ruído o valor de $85 \mathrm{~dB}$ (A) para uma jornada diária de 8 horas. Já para vibração de corpo inteiro (VCI) o anexo 8 da referida norma, apresenta como limite de exposição (LE) diária o valor de aceleração resultante de exposição normalizada (aren) de $1,1 \mathrm{~m} \mathrm{~s}^{-2}$ e o valor da dose de vibração resultante (VDVR) de $21 \mathrm{~m} \mathrm{~s}^{1,75}$ (BRASIL, 2014). Segundo FUNDACENTRO (2013) o limite de exposição pode ser entendido como condições sobre as quais acredita-se que a maioria das pessoas possam estar expostas repetidamente sem sofrer efeitos adversos a saúde.

Ressalta-se que exposições a níveis elevados de ruído podem trazer efeitos auditivos e extra-auditivos ao organismo dos trabalhadores tais como: perda auditiva, distúrbios gastrintestinais, distúrbios no sistema nervoso proporcionando irritabilidade e nervosismo (SALIBA, 2013a). Ainda segundo o mesmo autor, a exposição a VCI pode trazer vários danos físicos e distúrbios ao organismo dos trabalhadores. Estudo epidemiológicos indicam que exposições constantes a níveis elevados de VCI podem resultar em danos na coluna vertebral, afetar o sistema circulatório e/ou urológico além do sistema nervoso central (SALIBA, 2013b; SALIBA, 2019). Observações de grupo de trabalhadores expostos a níveis elevados de VCI apontaram problemas na região dorsal e lombar, gastrointestinais, problemas nos discos intervertebrais e degeneração na coluna vertebral (SALIBA, 2013b; SALIBA, 2019)).

$\mathrm{Na}$ agricultura brasileira, estudos acadêmicos têm sido realizados com tratores agrícolas e seus implementos, avaliando-se separadamente os agentes de risco ruído (MAGALHÃES; CORTEZ; NAGAHAMA, 2012; BAESSO et al., 2017; SILVA et al., 2018; DAMASCENO et al., 2019; OLIVEIRA et al., 2020; FARIAS; SCHLOSSER, 2020; SILVA et al., 2021) e vibração (SANTOS et al., 2016; SANDI et al., 2018; SANDI et al., 2020) existindo, no entanto, poucos trabalhos que considerem a exposição simultânea a estes agentes de riscos ambientais, especialmente nos tratos culturais do cafeeiro. 
Segundo Fernandes e Morata (2002) a combinação de diferentes agentes estressores podem trazer uma série de efeitos negativos a saúde e bem estar dos trabalhadores. Sendo assim, a determinação concomitante dos níveis de ruído e vibração a que estão expostos operadores de conjuntos mecanizados, permite a realização de uma análise isolada e conjunta da exposição ocupacional.

Neste sentido, para que seja possível propor medidas de mitigação e controle a estes agentes de riscos, torna-se necessária a realização da sua quantificação e a compreensão do seu comportamento. Ainda neste contexto, Cunha, Duarte e Souza (2012) apontaram que é fundamental compreender os níveis de ruído e vibração dos equipamentos mecanizados para que seja possível apresentar possíveis estratégias de mitigação e controle das exposições.

Ressalta-se ainda, que a velocidade de operação é uma variável técnica de extrema importância para a aplicação de defensivos com conjuntos mecanizados e podem ter influência tanto nos níveis de vibração de corpo inteiro (VCI) quanto de ruído que chegam ao operador. Sendo assim, objetivou-se no presente estudo determinar o nível de vibração de corpo inteiro e ruído em um trator cafeeiro acoplado a um turbo pulverizador sob diferentes velocidades em ensaio padronizado. 


\section{MATERIAL E MÉTODO}

O experimento foi conduzido em lavoura cafeeira da Fazenda Escola do Instituto Federal de Educação, Ciência e Tecnologia do Sul de Minas (IFSULDEMINAS) - Campus Muzambinho - MG entre os meses de dezembro (2021) e janeiro (2022). Durante a realização do experimento a temperatura média registrada foi de $24,1^{\circ} \mathrm{C}$, umidade relativa $72,2 \%$ e a velocidade do vento de $1,2 \mathrm{~ms}^{-1}$.

A atividade foi desenvolvida em talhão cafeeiro, espaçamento 3,8 x 1,0 m, cultivar Catuaí Vermelho-144, com altura média das plantas de $2,3 \mathrm{~m}$, altitude $\pm 1.030 \mathrm{~m}$, apresentando, no momento da avaliação, o solo coberto com vegetação roçada e material orgânico em decomposição (Figura 1).

Figura 1: Cobertura do solo com vegetação roçada e material orgânico.

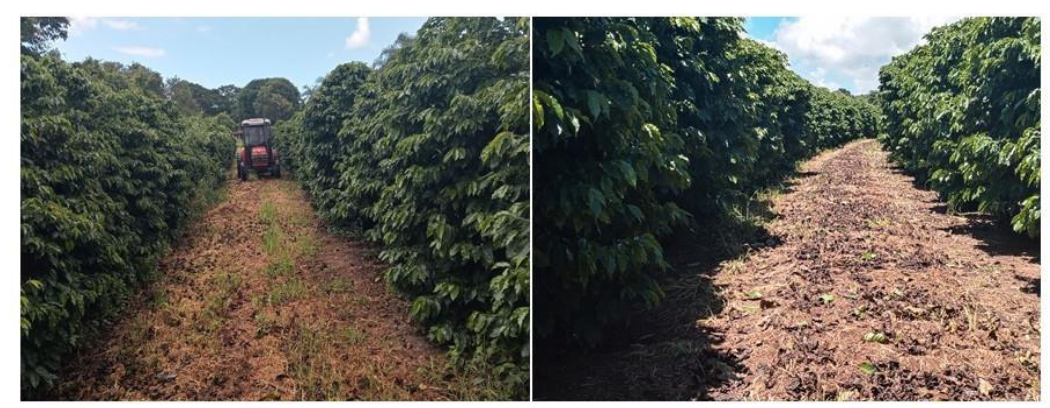

Fonte: Elaborado pelos autores (2022).

O conjunto mecanizado cafeeiro utilizado foi composto de um trator Yanmar Agritec 1155-4 SR, tração 4x4, potência $55(\mathrm{cv})$, peso total $2.280(\mathrm{Kg})$ utilizando pneus diagonais (dianteiros 700-18 e traseiros 14.9-24), lastro de $320 \mathrm{~kg}$ e acoplado ao implemento turbo pulverizador Jacto Arbus 400, com capacidade do reservatório de 400 L. O assento do operador é um banco POWERED 3 Y Yanmar.

Um único operador experiente fez a operação do conjunto mecanizado, sendo que a coleta dos dados teve início após a aprovação do Comitê de Ética em Pesquisas com seres humanos (CEP), processo $\mathrm{n}^{\mathrm{o}}$ 46207221.0.0000.8158.

O delineamento experimental utilizado foi o inteiramente casualizado (DIC), com o conjunto tratorizado devidamente abastecido com $400 \mathrm{~L}$ de água, funcionando com rotação do motor em 2200 rpm e deslocando-se em 4 diferentes velocidades de trabalho (V1, V2, V3 e V4), em rua de cafeeiro de $212 \mathrm{~m}$ de comprimento. As velocidades de trabalho foram: 2,3 $\mathrm{Km} \mathrm{h}^{-1}\left(2^{\mathrm{a}}\right.$ reduzida); 3,5 $\mathrm{Km} \mathrm{h}^{-1}$ ( $3^{\mathrm{a}}$ reduzida); 4,6 $\mathrm{Km} \mathrm{h}^{-1}$ (4 $4^{\mathrm{a}}$ reduzida); 5,8 $\mathrm{Km} \mathrm{h}^{-1}$ (1 $1^{\mathrm{a}}$ simples).

Para cada velocidade de trabalho foram realizadas 6 repetições, sendo que o tempo de coleta, foi aquele necessário para o conjunto tratorizado se deslocar na faixa de operação de 212 m na rua com plantas de cafeeiros. Em cada avaliação foram determinados o nível Vibração de corpo inteiro (VCI) e de ruído no posto de trabalho. 


\section{Vibração de Corpo Inteiro (VCI)}

A aquisição dos dados de VCI, ocorreu com medidor de vibração, marca $01 \mathrm{~dB}, \mathrm{n}^{\circ}$ série 10.648 , calibrado com certificado $\mathrm{RBC}$, atendendo-se aos requisitos de ponderação $\left(\mathrm{W}_{\mathrm{k}}\right)$ para $\mathrm{o}$ eixo longitudinal " $z$ " e $\left(\mathrm{W}_{\mathrm{d}}\right)$ para os eixos transversais " $\mathrm{x}$ " e " $\mathrm{y}$ " e as características previstas nas normas internacionais ISO 8041 (2005), ISO 2631-1 (1997) e norma de higiene ocupacional NHO 09. O conjunto de medição foi conectado ao transdutor triaxial, configurado segundos os parâmetros da NHO 09, para avaliações de VCI. Foram avaliados simultaneamente os eixos ortogonais " $\mathrm{x}$ ", " $y$ " e "z". O transdutor de corpo inteiro foi posicionado e fixado no banco do operador conforme estabelecido pela NHO 09 (FUNDACENTRO, 2013).

Após realização das avaliações, os dados coletados foram descarregados e processados utilizando-se o software dBMaestro 5.5. Conforme indicado por FUNDACENTRO (2013), foram determinadas as acelerações nos três sentidos perpendiculares, "x", "y" e "z" e o valor da aceleração média resultante (amr) foi obtido por meio da soma da raiz dos quadrados das acelerações médias expressa em $\mathrm{m} \mathrm{s}^{-2}$. Em seguida, determinou-se a aceleração resultante de exposição parcial ( $\left(\mathrm{arep}_{\mathrm{i}}\right)$ pela média aritmética das acelerações médias resultantes $\left(\mathrm{amr}_{\mathrm{ik}}\right)$ obtidas para repetição da exposição “i” expressa em ms², de acordo com a NHO 09 (2013). A aceleração resultante de exposição normalizada (aren) foi estimada e expressa em $\mathrm{m} \mathrm{s}^{-2}$ e o valor da dose de vibração resultante (VDVR) foi obtido a partir do método de dose de vibração à quarta potência, determinado na direção "j”, sendo que "j” corresponde aos eixos ortogonais "x", "y" ou "z", expresso em $\mathrm{m} \mathrm{s}^{-175}$. Esses parâmetros (aren e VDVR), foram estimados considerando-se as acelerações amr obtidas, para as diversas velocidades de ensaio, como representativas da exposição diária, ou seja, esses valores de ensaio para cada velocidade foram projetados para uma exposição diária de 8 horas. É importante observar que na prática, nas situações habituais/cotidianas de exposição ao agente, o aren e VDVR, são representativos da exposição dos trabalhadores à VCI presente na sua jornada diária. Desse modo, a estimativa realizada visou apenas mostrar a influencia da velocidade, nessa estimativa da exposição díaria, sem considerar outras variáveis que influenciam na exposição ocupacional ao agente. Ressalta-se que de acordo com a NR 15, para avaliações de VCI obrigatoriamente o empregador deve comprovar a avaliação tanto da aren quanto o VDVR (BRASIL, 2014).

Os valores obtidos foram comparados tanto com o limite de exposição (LE) da NR 15 e nível de ação da NR 9 quanto com os critérios de julgamento e tomada de decisão da NHO 09 (Tabela 1). 
Tabela 1: Critério de julgamento e tomada de decisão

\begin{tabular}{|c|c|c|c|}
\hline $\begin{array}{l}\text { aren* } \\
\left(\mathrm{m} \mathrm{s}^{-2}\right)\end{array}$ & $\begin{array}{l}\text { VDVR** } \\
\left(\mathrm{m} \mathrm{s}^{-1,75}\right)\end{array}$ & $\begin{array}{l}\text { Consideração } \\
\text { Técnica }\end{array}$ & Atuação \\
\hline 0 a 0,5 & 0 a 9,1 & aceitável & $\begin{array}{l}\text { No mínimo manutenção da condição } \\
\text { existente }\end{array}$ \\
\hline$>0,5$ a $<0,9$ & $>9,1$ a $<16,4$ & $\begin{array}{l}\text { acima do nível de } \\
\text { ação }\end{array}$ & $\begin{array}{l}\text { No mínimo adoção de medidas } \\
\text { preventivas }\end{array}$ \\
\hline 0,9 a 1,1 & 16,4 a 21 & região de incerteza & $\begin{array}{l}\text { Adoção de medidas preventivas e } \\
\text { corretivas visando à redução da } \\
\text { exposição diária }\end{array}$ \\
\hline Acima de 1,1 & Acima de 21 & $\begin{array}{l}\text { acima do limite de } \\
\text { exposição }\end{array}$ & $\begin{array}{l}\text { Adoção imediata de medidas } \\
\text { corretivas. }\end{array}$ \\
\hline
\end{tabular}

Legenda: *Aceleração resultante de exposição normalizada (aren); **Valor da dose de vibração resultante (VDVR). Fonte: Adaptado Norma de Higiene Ocupacional - NHO 09 (2013).

\section{Ruído Ocupacional}

As avaliações do ruído em condições de operação foi realizada utilizando-se medidor integrador de uso pessoal (dosímetro) marca Instrutherm, modelo DOS-1000, com filtro de banda de oitava, função datalloger, configurado com circuito de ponderação "A", circuito de resposta lenta (slow), critério de referência $85 \mathrm{~dB}$ (A), limiar de integração $80 \mathrm{~dB}$ (A), indicação de picos superiores a 115 dB (A) e incrementos de duplicação q-5 e q-3 de modo a atender simultaneamente os critérios da NR 15 e NHO 01.

$\mathrm{O}$ equipamento foi posicionado na zona auditiva do operador (raio de $150 \mathrm{~mm}$ ) medido a partir da entrada do canal auditivo. Os dados obtidos 1/1 oitava, foram descarregados e processados em software especifico (DOS-1000). Em seguida foi definido o nível de exposição normalizado (NEN) tanto para a NR 15 quanto para a NHO 01. Os valores obtidos foram comparados com o limite de exposição (LE) da NR 15, nível de ação NR 9.

\section{Estatística}

Foram realizadas a análise de regressão considerando-se a velocidade de trabalho e os níveis de aren e VDVR. Os modelos foram ajustados avaliando-se o erro percentual absoluto médio (MAPE,\%), e a precisão usando o coeficiente de determinação ajustado ( $\left.\mathrm{R}^{2}\right)$ (CORNELL; BERGER, 1987).

O conjunto de dados coletados foi submetido à análise de variância, por meio do teste $\mathrm{F}$. Nos casos em que o valor do teste $\mathrm{F}$ for significativo, foram realizados testes de comparação de médias de Tukey, ao nível de 5\% de significância de erro. Para tanto, foi empregado o software estatístico computacional "Sisvar" de Ferreira (2011). 


\section{RESULTADOS E DISCUSSÃO}

Os valores médios de vibração de corpo inteiro (VCI) obtidos em ensaio padronizado com conjunto mecanizado utilizado para aplicação de defensivos na cafeicultura, estão apresentados na Tabela 2. Os resultados demonstraram que os níveis de aceleração resultante de exposição normalizada (aren) e dose de vibração resultante (VDVR) aumentaram quando se empregou maiores velocidades de deslocamento (Tabela 2).

Tabela 2: Valores médios de vibração ocupacional de corpo inteiro (aren; VDVR) ( \pm desvio padrão) gerado por conjunto tratorizado utilizado para aplicação de defensivos na cafeicultura submetido a diferentes velocidades de trabalho

\begin{tabular}{ccc}
\hline $\begin{array}{c}\text { Velocidade } \\
\left(\mathrm{Km} \mathrm{h}^{-1}\right)\end{array}$ & $\begin{array}{c}\text { aren* } \\
\left(\mathrm{m} \mathrm{s}^{-2}\right)\end{array}$ & $\begin{array}{c}\text { VDVR** } \\
\left(\mathrm{m} \mathrm{s}^{-1,75}\right)\end{array}$ \\
\hline 5,8 & $0,78 \pm 0,06 \mathrm{a}$ & $11,68 \pm 1,01 \mathrm{a}$ \\
4,6 & $0,62 \pm 0,01 \mathrm{~b}$ & $9,71 \pm 0,84 \mathrm{~b}$ \\
3,5 & $0,48 \pm 0,07 \mathrm{c}$ & $6,84 \pm 1,50 \mathrm{c}$ \\
2,3 & $0,32 \pm 0,03 \mathrm{~d}$ & $5,29 \pm 0,88 \mathrm{c}$ \\
\hline $\mathrm{C} . \mathrm{V}(\%)^{(1)}$ & 8,60 & 13,01
\end{tabular}

Legenda: *Aceleração resultante de exposição normalizada; **Valor da dose de vibração resultante; ${ }^{(1)}$ Coeficiente de variação em porcentagem; Médias seguidas de mesma letra minúscula nas colunas não diferem entre si pelo teste de Tukey ao nível de 5\% de significância. Fonte: Elaborado pelos autores (2022).

Na menor velocidade empregada $\left(2,3 \mathrm{Km} \mathrm{h}^{-1}\right)$ o aren foi de $0,32 \mathrm{~ms}^{-2}$ e o VDVR 5,29 $\mathrm{ms}^{-}$ ${ }^{1,75}$, enquanto que na velocidade de $5,8 \mathrm{Km} \mathrm{h}^{-1} \mathrm{o}$ aren foi de $0,78 \mathrm{~ms}^{-2}$ e o VDVR 11,68 $\mathrm{ms}^{-2}$ (Tabela 1), indicando um aumento de $143,7 \%$ na aren e $120,7 \%$ para o VDVR. Os modelos lineares ajustados (aren e VDVR) em função da velocidade de deslocamento estão apresentados na Figura 2. Pode-se afirmar que os modelos foram acurados com valores de $\mathrm{R}^{2}$ ajustado de 0,93 para aren e 0,84 para o VDVR (Figura 2).

Figura 2: Modelos lineares ajustados para estimativa da aceleração resultante de exposição normalizada e valor da dose de vibração resultante em função da velocidade de deslocamento. 
A)

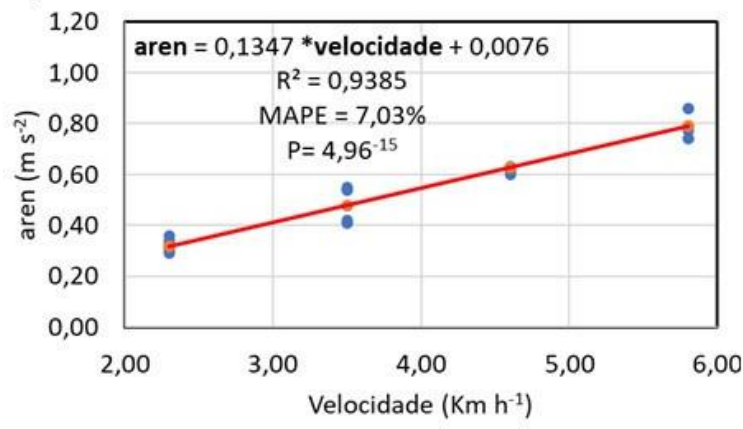

B)

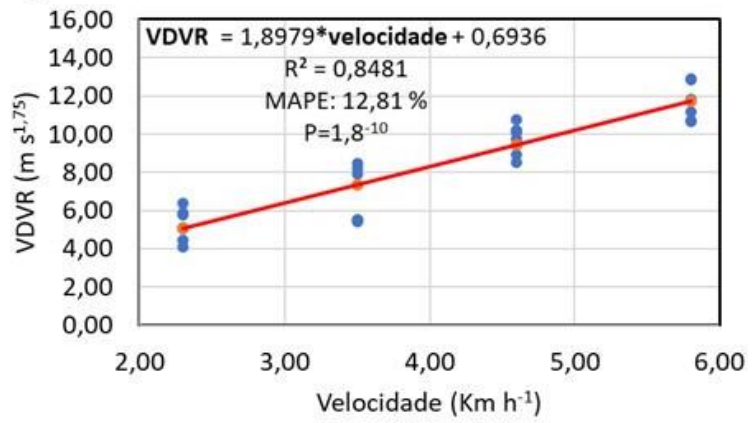

Legenda: Aceleração resultante de exposição normalizada (A) e Valor da Dose de Vibração Resultante (B). Fonte: Elaborado pelos autores (2022).

Portanto, a velocidade de operação influenciou diretamente nos níveis de VCI quantificados no presente estudo, indicando que a escolha da velocidade de trabalho deve ser considerada como estratégia positiva para a prevenção de danos causados à saúde de trabalhadores expostos a VCI. Sandi et al (2020) também evidenciaram em seus estudos que as maiores velocidades de operação influenciaram diretamente para aumentar as acelerações incidentes sobre o corpo inteiro dos trabalhadores. Resultados semelhantes foram apontados por Santos et al (2014), demonstrando que o aumento da vibração ocorreu de maneira constante, a partir do aumento da velocidade.

Muito embora os valores de VCI estejam abaixo do limite de exposição (aren 1,1 $\mathrm{m} \mathrm{s}^{-2}$; VDVR $21 \mathrm{~m} \mathrm{~s}^{-1,75}$ ) para uma jornada de trabalho de 8 horas conforme preconiza a a NR 15 (BRASIL, 2014), tanto a NHO 9, quanto a NR 9 estabelecem que as condições que ultrapassem o nível de ação (aren 0,5 $\mathrm{m} \mathrm{s}^{-2}$; VDVR 9,1 $\mathrm{m} \mathrm{s}^{-1,75}$ ) devem ser objeto de controle sistemático e devem ser adotadas medidas preventivas de modo a evitar que que as exposições tragam danos a saúde dos trabalhadores expostos e que o limite de exposição seja superado (BRASIL, 2021; FUNDACENTRO, 2013).

No presente estudo, pode-se observar que nas velocidades de 5,8 e 4,6 $\mathrm{Km} \mathrm{h}^{-1}$ os níveis de VCI ultrapassaram o nível de ação estabelecidos pela NR 9 (Tabela 2; Figura 2) tanto de aren $\left(0,5 \mathrm{~m} \mathrm{~s}^{-2}\right)$ quanto de VDVR $\left(9,1 \mathrm{~m} \mathrm{~s}^{-1,75}\right)$. Santos et al (2016) realizando avaliação da exposição do operador de trator à vibração na operação de gradagem, também encontraram valores acima do nível de ação para todas as condições avaliadas, sendo que à medida que a velocidade e rotação aumentaram, as VCI também aumentaram.

Portanto, de acordo com o critério de julgamento e tomada de decisão da NHO 09, nas velocidades 5,8 e 4,6 $\mathrm{Km} \mathrm{h}^{-1}$ torna-se necessário no mínimo a adoção de medidas preventivas. Estas medidas devem incluir o monitoramento periódico da exposição, informação e orientação aos trabalhadores e o controle médico (FUNDACENTRO, 2013). 
Em relação aos níveis de ruído, pode-se observar que não houve diferença significativa na interação entre os fatores velocidade de deslocamento (V1, V2, V3 e V4) e os resultados do ruído, segundo os critérios técnicos utilizados para avaliação (NHO e NR 15) ( $\mathrm{P}=0,9860 ; p>0,05)$ (Tabela 3). A não existência de diferença significativa no nível de ruído, pode estar relacionada ao fato que, independentemente da velocidade selecionada este conjunto mecanizado utilizou a rotação constante do motor de $2.200 \mathrm{rpm}$ para alcançar $540 \mathrm{rpm}$ na tomada de força e potência (TDP), resultando num ruído que supera àquele gerado em razão da variação de velocidade do trator.

Tabela 3: Nível médio de ruído ocupacional ( \pm desvio padrão) de conjunto tratorizado utilizado para aplicação de defensivos na cafeicultura submetido a diferentes velocidades de trabalho e avaliado segundo a NR 15 e NHO 01

\begin{tabular}{ccc}
\hline $\begin{array}{c}\text { Velocidade } \\
\left(\mathrm{Km} \mathrm{h}^{-1}\right)\end{array}$ & NR 15 & NHO 01 \\
\hline 5,8 & \multicolumn{2}{c}{$(\mathrm{NEN}-\mathrm{dB}-\mathrm{A})$} \\
4,6 & $90,3 \pm 0,65$ & $90,3 \pm 0,62$ \\
3,5 & $90,4 \pm 0,32$ & $90,3 \pm 0,34$ \\
2,3 & $91,0 \pm 0,69$ & $91,1 \pm 0,72$ \\
C.V $(\%)$ & $90,9 \pm 0,37$ & $91,0 \pm 0,39$ \\
\hline
\end{tabular}

Legenda: *Nível de Ruído Ocupacional/Nível de Exposição Normalizado (NEN) em dB (A); ${ }^{(1)}$ Coeficiente de variação em \%; Fonte: Elaborado pelos autores (2022).

Ressalta-se que para todas as condições, os níveis médios de ruído encontrados estão acima do limite de exposição de 85 dB (A) para uma jornada de trabalho de 8 horas conforme determina a NR 15 (Tabela 3). Santos et al (2014) realizando avaliação dos níveis de ruído e vibração em um conjunto trator pulverizador também encontraram níveis superiores ao limite de exposição de $85 \mathrm{~dB}(\mathrm{~A})$. No estudo os autores relataram valores entre 97,4 e 98,2 dB (A), superiores aos encontrados neste ensaio 90,3 e 91,1 dB (A). A diferença entre os valores encontrados no presente estudo e os informados por Santos et al (2014) pode estar relacionada as diferenças técnicas entre os conjuntos mecanizados utilizados e rotação de operação.

Sendo assim, ao considerarmos o maior nível de ruído quantificado (91,1 dB (A)), o tempo de trabalho permitido sem proteção para que o limite de exposição diário não seja superado, será de aproximadamente 3 h e 30 minutos segundo a NR 15 e $2 \mathrm{~h}$ seguindo os com os critérios de duplicação q-3 da NHO 01. Destaca-se, no entanto que, o limite de exposição não deve ser considerado com linha divisória definitiva entre aqueles considerados seguros e inseguros, uma vez que este parâmetro ocupacional representa as condições sobre as quais acredita-se que a 
maioria dos trabalhadores possam estar expostos repetidamente sem sofrer efeitos adversos a saúde (FUNDACENTRO, 2001; ACGIH, 2018).

Ainda como estratégia preventiva de controle do ruído, recomenda-se a realização constante de manutenção preventiva, preditiva e se for o caso corretiva, uma vez que, a falta de manutenção pode contribuir para desequilíbrio dos componentes mecânicos que pode contribuir para elevar tanto o nível de ruído, quanto de VCI. Silva et al (2021) destacam que a falta de manutenção e lubrificação dos conjuntos mecânicos podem favorecer o aumento do nível de ruído.

Os níveis de ruído encontrados no presente estudo também reforçam a necessidade do monitoramento da saúde auditiva de operadores de conjuntos mecanizados na agricultura. No Brasil, a NR 7 que trata do programa de controle médico e saúde ocupacional (PCMSO) anexo II estabelece que devem ser submetidos a exames audiométricos todos os trabalhadores que estejam expostos em atividades com nível de pressão sonora (NPS) superiores ao nível de ação (80 dB (A)), independentemente do uso de protetor auricular (BRASIL, 2020).

No caso do ruído, exposições superiores ao limite podem implicar na necessidade de utilização de protetores auricular com nível de atenuação adequado. A variação do nível de ruído quantificado dentro do espectro de frequência 1/1 oitava $(63$ a $8000 \mathrm{~Hz})$ para cada velocidade de trabalho selecionada (Figura 3).

Figura 3: Espectro de frequência do nível de pressão Sonora (NPS) por banda de 1/1 oitava para as diferentes velocidades de trabalho

A)

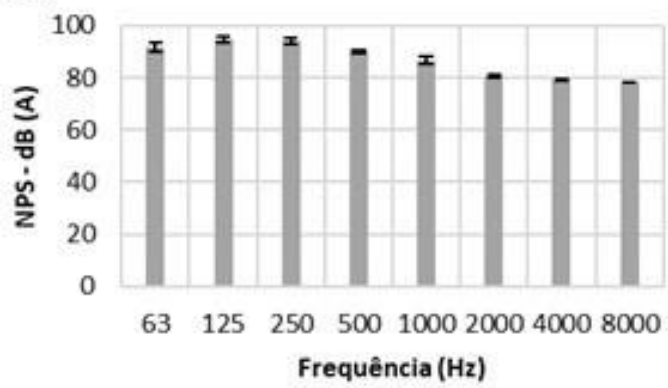

C)

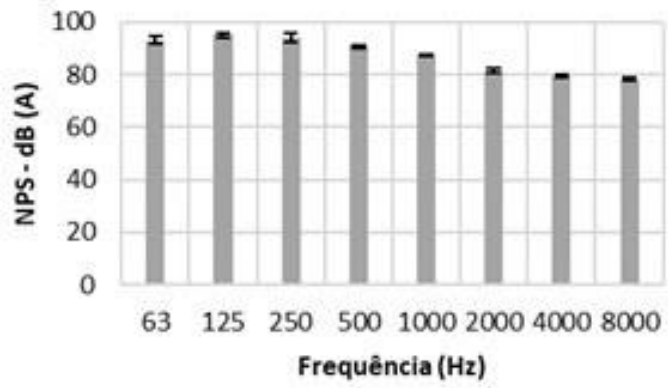

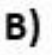

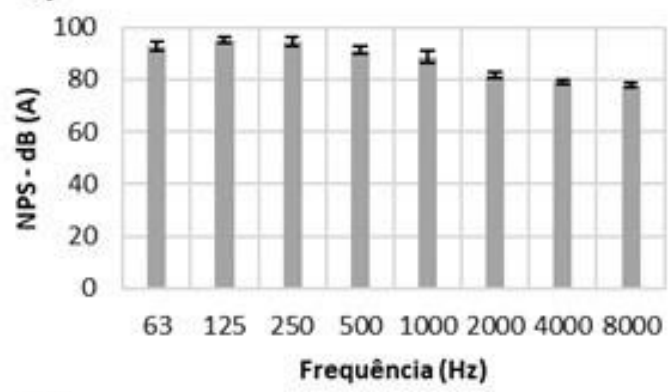

D)

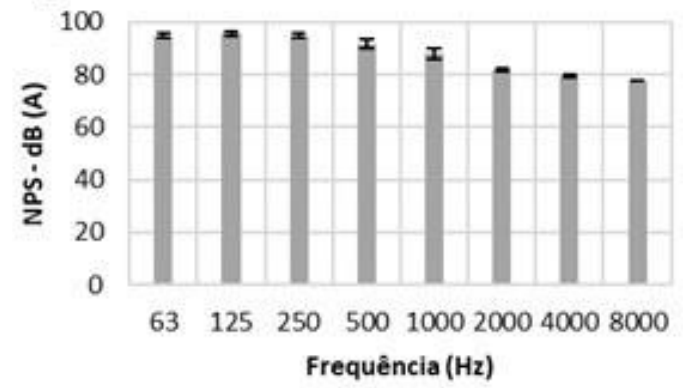


Legenda: V1 - 2,3 $\mathrm{Km} \mathrm{h}^{-1} \mathrm{Km}(\mathrm{A})$; V2 - 3,5 $\mathrm{Km} \mathrm{h}^{-1}(\mathrm{~B})$; V3 - 4,6 $\mathrm{Km} \mathrm{h}^{-1}$ (C); V4 - 5,8 $\mathrm{Km} \mathrm{h}^{-1}$ (D). Fonte: Elaborado pelos autores (2022).

Pode-se observar que os maiores níveis de ruído foram obtidos nas frequências de 125 e $250 \mathrm{~Hz}$ com valores próximos de 94 a $95 \mathrm{~dB}$ (A) e os menores nas frequências de 2000 a 8000 $\mathrm{Hz}$ com valores entre 80 e $78 \mathrm{~dB}(\mathrm{~A})$. Destaca-se ainda que a análise do espectro de frequência demonstrou que nas frequências superiores a $2000 \mathrm{~Hz}$ os níveis de ruído ficaram abaixo de $80 \mathrm{~dB}$ (A) (Figura 3).

A seleção de proteção auditiva deve considerar protetores cujos níveis de atenuação sejam adequados tecnicamente ao risco e ao espectro de frequência presente no ambiente de trabalho. Ressalta-se que de acordo com a ABNT (2021) NBR 16.077 a estimativa do nível de exposição com proteção pode ser calculada por dois métodos sendo: método simplificado e método longo. Segundo Saliba (2013a) a utilização do método longo/detalhado apresenta nível de confiança estatística de $98 \%$, enquanto que o método tradicional (direto) possui nível de confiança de $84 \%$

\section{CONCLUSÃO}

O aumento da velocidade contribuiu para elevar os níveis de VCI, mas não influenciou significativamente para nos níveis de ruído ocupacional.

Nas velocidades de 4,6 e 5,8 $\mathrm{Km} \mathrm{h}^{-1}$ os valores de aceleração resultante de exposição normalizada (aren) e valor da dose de vibração resultante (VDVR) ultrapassaram o nível de ação (aren 0,5 $\mathrm{m} \mathrm{s}^{-2}$; VDVR 9,1 $\mathrm{m} \mathrm{s}^{-1,75}$ ), mas ficaram abaixo do limite de exposição determinado pela NR 15 (aren 1,1 $\mathrm{m} \mathrm{s}^{-2}$; VDVR $21 \mathrm{~m} \mathrm{~s}^{-1,75}$ ).

Em todas as velocidades avaliadas o nível de ruído ficou acima do limite de exposição de $85 \mathrm{~dB}$ (A) para uma jornada de trabalho diária de 8 horas evidenciando a necessidade de adoção de estratégias preventivas e corretivas.

\section{AGRADECIMENTO}

Agradecemos a Universidade Estadual Paulista "Julio de Mesquita Filho" - UNESP, campus Tupã - SP pelo desenvolvimento do estágio Pós Doutorado (Processo n. 3318).

\section{REFERENCIAS}

AMERICAN CONFERENCE OF GOVERNMENTAL INDUSTRIAL HYGIENIST ACGIH. TLVs e BEIs: baseado na documentação dos Limites de Exposição Ocupacional (TLVs) para Substâncias Químicas, Agentes Físicos e Índices 
Biológicos (BEIs). Tradução Associação Brasileira de Higienistas Ocupacionais ABHO. São Paulo, 2018. p.298.

ASSOCIAÇÃO BRASILEIRA DE NORMAS TÉCNICAS - ABNT. NBR 16077: Equipamento de proteção individual - Protetores auditivos - Método de cálculo do nível de pressão sonora na orelha protegida. Rio de Janeiro, 2021. 10p.

BAESSO, M. M.; MODOTO, A. J.; BAESSO, R. C. E.; FISCHER, C. Níveis de Ruído Emitidos por Tratores Agrícolas. Brazilian Journal of Byosystems Engineering, v. 11, n. 3, p. 229-238, 2017.

BRASIL. Ministério da Economia - Secretaria Especial de Previdência e Trabalho. Portaria n ${ }^{\circ} 6.735$ de 10 de março de 2020. NR 09 - Avaliação e Controle das Exposições Ocupacionais a agentes Físicos, Químicos e Biológicos. Diário Oficial da União, seção 1, Brasilia, DF, mar. 2020. Disponível em: https://enit.trabalho.gov.br. Acesso em: 17 de Jan. 2022.

BRASIL. Ministério do Trabalho e Emprego. Portaria n 1297 de 13 de Agosto de 2014. Aprova o Anexo I - Vibração da Norma Regulamentadora no 9 - Programa de Prevenção de Riscos Ambientais (PPRA), altera o anexo 8 - Vibração da Norma Regulamentadora $\mathbf{n}^{\mathbf{0}} 15$ - Atividades e Operações Insalubres, e da outras providencias. Diário Oficial da União. Brasília, 2014.

BRASIL. Ministério da Economia - Secretaria Especial de Previdência e Trabalho. Portaria no 6.735 de 10 de março de 2020. Diário Oficial da União, seção 1, Brasilia, DF, mar. 2020. Disponível em: https://enit.trabalho.gov.br. Acesso em: 17 de jan. 2022.

CUNHA, J. P. A. R da.; DUARTE, M. A. V.; SOUZA, C. M. A de. Vibração e ruído emitidos por dois tratores agrícolas. Revista IDESIA (Chile), v. 30, n. 1, p. 25-34, 2012.

DAMASCENO, F. A.; SOARES, C. M.; OlIVEIRA, C. E. A.; FERRAZ, G. A.S.; SARAZ, A. O. Avaliação do Nível de Ruído Emitido por um Trator Agrícola Acoplado a uma Colhedora de Milho. Revista Engenharia na Agricultura, v. 27, n. 5, p. 412-419, 2019.

FARIAS, M. S de.; SCHLOSSER, J. F. Níveis de Ruído no Posto de Operação de um Trator Agrícola na Operação de Semeadura. Tecno-lógica, Santa Cruz do Sul, v. 24, n. 1, p. 47-52, 2020.

FERNANDES, M.; MORATA, T. C. Estudo dos efeitos auditivos e extra-auditivos da exposição ocupacional a ruído e vibração. Revista Brasileira Otorrinolaringologia. v. 68, n. 5, p. 705-713, 2002.

FUNDAÇÃO JORGE DUPRAT FIGUEIREDO DE SEGURANÇA E MEDICINA DO TRABALHO - FUNDACENTRO. Norma de Higiene Ocupacional - Procedimento Técnico. Avaliação da exposição ocupacional a vibrações de corpo inteiro. NHO 09. São Paulo, 2013. 63 p.

LEITE, D. M.; FERNANDES, H. C.; FURTADO JUNIOR, M. R.; FEITOSA, J. R.; SANTOS, D. W. F do. N. Caracterização das vibrações dos postos de operação de um trator agrícola em condição de tração. Brazilian Journal of Animal and Environmental Research. Curitiba, v. 3, n. 4, p. 3808-3821, 2020. 
MAGALHÃES, A. T.; CORTEZ, J. W.; NAGAHAMA, H de J. Nível de Ruído de um Trator Agrícola em Função da Rotação, da Distância, da Velocidade e da Condição do Solo Obtido por Meio de Decibelimetro com e sem Protetor de Ventto. Engenharia Agrícola, Botucatu, v. 27. N. 4, p. 27-44, 2012.

OLIVEIRA, G. S. A.; OLIVEIRA JUNIOR, G. G de.; REZENDE, R. N.; PAULA, L. E de. R.; SILVA, J. A.; RAMIRIO, L. D.; SILVA, A. B da.; COUTINHO, P. R do. V. Variabilidade Espacial do Nível de Ruído em um Conjunto Trator-Pulverizador sob Diferentes Rotações. Brazilian Journal of Development. Curitiba, v. 6, n. 11, p. 8609486101, 2020.

SALIBA, T. M. Manual Prático de Higiene Ocupacional e PPRA: avaliação e controle dos riscos ambientais. $4^{\mathrm{a}}$. ed. São Paulo : LTr, 2013a.367 p.

SAliBA, T. M. Manual Prático de Avaliação e Controle de Vibração - PPRA. $2^{\mathrm{a}}$. Ed. São Paulo: LTr, 2013b, 101 p.

SALIBA, T. M. Manual Prático de Avaliação e Controle de Vibração - PPRA. $6^{\text {a }}$. Ed. São Paulo: LTr, 2019, 119 p.

SANDI, J.; TESTA, J. V. P.; MARTINS, M. B.; FIORESE, D. A.; LANÇAS, K. P. Vibração ocorrente sobre o corpo inteiro do operador de trator agrícola em ensaio padronizado. Revista de Agricultura Neotropical, Cassilândia-MS, v. 5, n. 2, p. 54-60, abr./jun. 2018.

SANDI, J.; MARTINS, M. B.; AMORIM, D. J.; SANTOS, J. E. G dos.; LANÇAS, K. P. Avaliação da Vibração Incidente sobre o Operador de Trator Agrícola em Diferentes Condições Superficiais. Energia na Agricultura, Botucatu, v. 35, n. 3, p. 308-316, 2020.

SANTOS, L. N. dos.; FERNANDES, H. C.; SOUZA, A. P de.; FURTADO JUNIOR, M. R.; SILVA, R. M. F. Engenharia na Agricultura, Viçosa, v. 22, n. 2, p. 112 - 118, 2014.

SANTOS, V. C dos.; MONTEIRO, L de. A.; MACEDO, D. X. S.; MELO, R. P.; QUEIROZ, M. L de. V. Avaliação da Exposição do Operador à Vibração na Operação de Gradagem. Energia na Agricultura, Botucatu, v, 31, n. 4, p. 312-316, 2016.

SILVA, J. A. R da.; OLIVEIRA JUNIOR, G. G de.; COSTA, C. E de. M.; SILVA, A. B da.; GABRIEL, C. P. C.; PUTTI, F. F. Occupacional Noise Level In Mechanized and Semimecanized Harvest of Coffee Fruits. Coffee Science, Lavras, v. 13, n. 4, p. 448-454, 2018.

SILVA, M. A da.; OLIVEIRA JUNIOR, G. G de.; APARECIDO, L. E de. O.; CUNHA, I de. A.; SILVA, J. A. R da. Dispersão do Ruído Gerado por um Conjunto Tratorizado Cafeeiro em Condição Estática: Estudo de Caso entre Diferentes Posições e Distâncias. Brazillian Journal of Development, Curitiba, v. 7, n. 4, p. 37500-37511, 2021. 